\title{
Cross-correlating spectroscopic and photometric galaxy surveys
}

\author{
Martin B. Eriksen ${ }^{1}$ and Enrique Gaztañaga ${ }^{2}$ \\ ${ }^{1}$ Sterrewacht Leiden, University of Leiden, \\ NL-2333 CA, Leiden, the Netherlands \\ email: marberi@strw.leidenuniv.nl \\ ${ }^{2}$ Institut de Ciencies de l'Espai (ICE-IEEC/CSIC), Campus UAB \\ 08193 Bellaterra, Spain \\ email: gazta@ice.cat
}

\begin{abstract}
Does photometric and spectroscopic survey benefit from overlapping areas? The photometric survey measures 2D Weak Lensing (WL) information from galaxy shape distortions. On the other hand, the higher redshift precision of an spectroscopic survey allows measurements of redshift space distortions (RSD) and baryonic accustic oscillations (BAO) from 3D galaxy counts.

The two surveys are combined using 2D-correlations, using sufficiently narrow bins to capture the radial information. This poster present effects of RSD and intrinsic correlations between narrow redshift bins. In understanding how the effects affects cosmological constrains, we first define two stage-IV and then present forecast for various configurations. When surveys overlap, they benefit from additional cross-correlations and sample variance cancellations from overlapping volumes. For a combined dark energy and growth history figure of merit, the result increase $50 \%$ for overlapping surveys, corresponding to $30 \%$ larger area.
\end{abstract}

Keywords. galaxies: distances and redshifts, techniques: spectroscopic surveys

\section{Introduction}

Galaxy surveys provide important insight to properties of dark matter and modified gravity. Photometric surveys target using galaxy shapes to measure weak gravitational lensing, while spectroscopic surveys can probe redshift space distortions (RSD) and baryonic acoustic oscillations (BAO) from excellent redshift information. The probes are powerful separate, but the combination can break parameter degeneracies. In addition the overlapping surveys allow for cross-correlating the observable. The benefit has been investigated by several groups Bernstein \& Cai (2011); Gaztañaga et al. (2012); Cai \& Bernstein (2012); Kirk et al. (2013); Font-Ribera et al. (2013); de Putter et al. (2013), but with large disagreement. Here we present our results, which is is a subset of a series of articles (in preparation) dealing with the modeling (paper-I), forecasts (paper-II), galaxy bias (paper-III) and the same-sky benefit (same-sky paper).

\section{Modeling}

Overdensities of galaxies can be expressed on the form

$$
\delta=\delta_{I}+\delta_{r}+\delta_{\kappa}
$$

where $\delta_{I}$ is the intrinsic overdensity, $\delta_{r}$ is the redshift space contribution and $\delta_{\kappa}$ is the weak lensing magnification. In this work, we forecast the constraint using crosscorrelations in narrow redshift bins. The narrow bins capture the bulk of information 

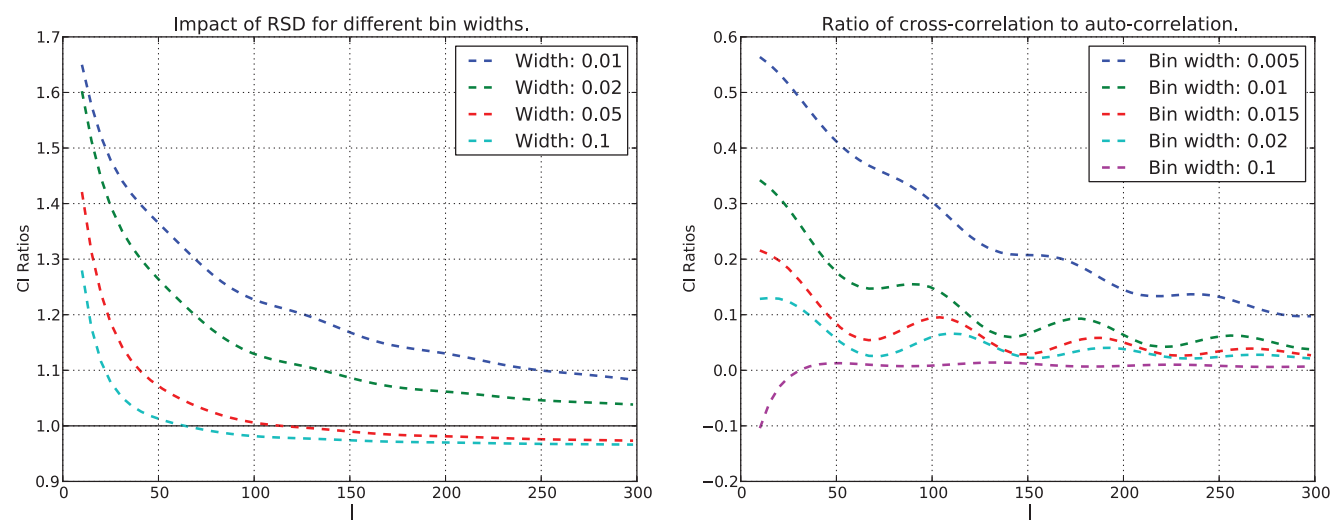

Figure 1. This figure who the effect of RSD and intrinsic correlations for different narrow bins. The left panel show the redshift to real space ratio for an auto-correlation at $z=0.5$. The right panel shows the ratio of a cross-correlation between an adjacent bin and the auto-correlation, with the first bin starting at $z=0.5$.

\begin{tabular}{l|r|r}
\hline Parameter & Photometric (F) & Spectroscopic (B) \\
\hline Area [sq.deg] & 14000 & 14000 \\
Redshift uncertainty & $0.05(1+\mathrm{z})$ & $0.001(1+\mathrm{z})$ \\
Magnitude limit & $\mathrm{i}<24.1$ & $\mathrm{i}<22.5$ \\
Bin width & $0.07(1+\mathrm{z})$ & $0.01(1+\mathrm{z})$ \\
Density [gal/sq.arcmin] & 6.5 & 0.4 \\
\hline
\end{tabular}

Table 1. The photometric and spectroscopic survey are modeled as two separate galaxy populations, with a brief description given in the table. The redshift uncertainty given is the Gaussian dispersion and both samples are magnitude limited. For redshift binning, the spectroscopic sample is analyzed using 10 times thinner bins, to properly include radial information.

Asorey et al. (2012), while simplifying the covariance between the surveys and the inclusion of redshift uncertainties.

\section{Redshift Space Distortions (2D) and intrinsic cross-correlations.}

Fig. 1 show two effects for narrow redshift bins. Decreasing the redshift bin will increase the RSD effect in the auto-correlations. At $l=10, z=0.5$ and $\Delta z=0.01$, the RSD increase the signal with $60 \%$. In addition the linear RSD effect is reaching smaller scales for thinner bins. The right panel show a substantial signal of cross-correlations between adjacent redshift bins. These cross-correlations has sufficient signal-to-noise (paper-I) and contribute significantly to the combined constraints (paper-II). Since this effect is zero in narrow redshift bins, it requires performing the exact calculations.

\section{Forecasts}

\subsection{Assumptions}

The Fisher matrix forecast use 2D-correlations in Fourier space (Cls) as observable and model the two surveys as separate galaxy populations (see Table 1). Observable are limited $l<=300$, with additional cuts on low redshift to remove non-linear scales (paperII) and the fiducial parameters are equal to the MICE simulations (Fosalba et al. 2008). To investigate the benefit of overlap, we define

$$
\mathrm{FoM}_{S} \equiv \frac{1}{\operatorname{det}\left[F_{S}^{-1}\right]}
$$




\begin{tabular}{lrrrrr}
\hline $10^{-3}$ FoM $\gamma w$ & Fiducial & fixBias & NoLens & NoRSD & NoBAO \\
\hline FxB-All & 31.5 & 189 & 5.86 & 14.7 & 21.7 \\
F+B-All & 20.8 & 157 & 4.69 & 9.22 & 13.3 \\
Improvement & 1.5 & 1.2 & 1.2 & 1.6 & 1.6 \\
\hline F-All & 2.55 & 38.4 & 0.031 & 2.13 & 1.95 \\
B-All & 6.71 & 44.1 & 4.14 & 2.46 & 4.27 \\
\hline Improvement & 1.2 & 0.99 & 1.1 & 1.1 & 3.4 \\
\hline
\end{tabular}

\begin{tabular}{lrrrrr} 
FoM $\gamma$ & Fiducial & xBias & No Lens & No RSD & No BAO \\
\hline \hline FxB-Counts & 43 & 150 & 38 & 15 & 43 \\
F+B-Counts & 35 & 152 & 34 & 4.3 & 34 \\
\hline Improvement & 1.2 & 0.99 & 1.1 & 3.4 & 1.3 \\
\hline
\end{tabular}

Table 2. Subset of the forecast. The rows marked "All" includes galaxy counts and shear, while "Counts" only include galaxy counts. Here FxB and F+B respectively denote combining the surveys on overlapping and separate areas. On the columns, fiducial is the normal forecast, "xBias" fix the galaxy bias, "noLens" does not include weak lensing, "noRSD" perform all calculations in real space, while "noBAO" disable the BAO in the Eisenstein-Hu power spectrum. All numbers include Planck priors.

which extends the dark energy task force (DETF) FoM. The $F_{o} M_{w \gamma}$ with $S=w 0, w a, \gamma$ consider both dark energy and growth of structure, while $F o M_{\gamma}$ with $S=\gamma$ only focus on the growth. When estimating the FoMs, we marginalize over the DETF parameters and the galaxy bias. The bias model is scale-independent and depend linearly on the redshift. For each population, the bias in each bin is treated as a separate variable and without prior knowledge.

\subsection{Results}

Table 2 show as subset of the forecast results (paper-II). Weak lensing is the most important contribution (FxB-All), with RSD and BAO also contributing significantly. In the forecast, the overlapping surveys increasing the $\mathrm{FoM}_{w \gamma}$ with $50 \%$, corresponding to $30 \%$ larger area. Comparing the first and second column, the benefit is stronger for an unknown bias, but is still present when fixing the bias. The benefit comes from additional cross-correlations and sample variance cancellation (paper-II and same-sky paper). In addition, the same-sky ratio is higher without RSD or BAO, because they constrains parameter combinations where the overlap otherwise would contribute. This highlights the importance of including all effects in the combined forecast.

\section{References}

Asorey, J., Crocce, M., Gaztañaga, E., \& Lewis, A. 2012, MNRAS, 427, 1891

Bernstein, G. M. \& Cai, Y.-C. 2011, MNRAS, 416, 3009

Cai, Y.-C. \& Bernstein, G. 2012, MNRAS, 422, 1045

de Putter, R., Doré, O., \& Takada M. 2013, ArXiv e-prints

Font-Ribera, A., McDonald, P., Mostek, N., Reid, B. A., Seo, H.-J., \& Slosar, A. 2013, ArXiv e-prints

Fosalba, P., Gaztañaga, E., Castander, F. J., \& Manera, M. 2008, MNRAS, 391, 435

Gaztañaga, E., Eriksen, M., \& Crocce, e. 2012, MNRAS, 422, 2904

Kirk, D., Lahav, O., Bridle, S., Jouvel, S., Abdalla, F. B., \& Frieman, J. A. 2013, ArXiv e-prints 